\title{
Consommer pendant les périodes de transition de vie : Comment les expériences de service impactent les consommateurs en deuil?
}

\author{
Samuel GUILLEMOT, Univ Brest, LEGO, Quimper, France \\ Cécile PLAUD, ENSTA Bretagne, Brest, France
}

\begin{abstract}
Résumé : Les identités personnelles et sociales des consommateurs s'expriment lors des expériences de service, notamment lors des interactions avec le personnel en contact. Pourtant très peu de recherches s'intéressent à la perception de ces expériences lors des transitions de vie. En s'appuyant sur l'analyse du discours de 28 consommateurs en deuil, cette recherche identifie quatre éléments des expériences de service qui ont un impact positif et/ou négatif sur le stress perçu et la transition de rôle social : (a) le discours explicatif et (b) émotionnel du personnel en contact ; (c) la coordination des interfaces de services ; (d) et la relations aux autres consommateurs. Ces éléments sont discutés et des préconisations managériales à destination des prestataires de service sont proposées.
\end{abstract}

Mots-clés : Expériences de service - Transition de vie - interactions - personnel en contact

To cite: guillemot S. and Plaud C. (2019), Consommer Pendant les Periodes de tRansition de Vie : Comment les EXPERIENCES DE SERVICE IMPACTENT LES CONSOMMATEURS EN DEUIL ?, RECHERCHES EN SCIENCES DE GESTION, 130 (1), $61-83$

"Nous constatons que votre règlement a été rejeté par votre banque pour le motif suivant : titulaire décédé [...]. Nous vous mettons en demeure de régler cette créance sous dix jours, échéance à laquelle nous procéderons au recouvrement de la dette par voie judiciaire." $\mathrm{Ce}$ courrier adressé par un opérateur de téléphonie a choqué la famille d'une personne décédée. Elle ne comprend pas cette méthode du prestataire, alors même que le motif est clairement exposé par la banque. Cet exemple interpelle sur les effets néfastes d'une forte standardisation des services qui se révèlent inadaptés aux consommateurs vivant des transitions de vie. Les transitions de vie font référence aux événements qui entraînent un changement notoire dans la vie (la naissance d'un enfant, la mise en retraite, le décès d'un proche, etc.). L'individu perd alors ses repères, et entre dans une période de transition qui génère du stress et nécessite une adaptation afin d'établir un nouvel équilibre (Goguel d'Allondas, 2002). L'individu va voir évoluer son identité, ce qui va impacter ses préférences, attentes et schémas de consommation (Andreasen, 1984). La littérature sur la consommation en période de transition de vie se focalise principalement sur le rôle symbolique joué par un produit, une marque ou une pratique de consommation pour soutenir l'individu dans cette étape de son existence. Les consommations de services sont globalement absentes de cette littérature, alors que les identités personnelles et sociales des consommateurs - impactées lors des transitions de vie sont mobilisées lors des expériences de service (Goodwin et Gentry, 1997). Par exemple 70\% des veuves et veufs changent de conseillers financiers dans les trois années qui suivent le décès du conjoint car celui-ci n'arrive pas à établir une relation avec la personne survivante (Florian, 2011).

Cet article propose dès lors d'investiguer les liens entre les transitions de vie et les expériences de service. Avec des modes de vie de plus en plus mouvants et fragmentés, les consommateurs sont susceptibles de vivre des transitions de vie importantes et de plus en plus nombreuses (déménagement, divorce, recomposition familiale, changement professionnel, maladie invalidante, deuil du conjoint, etc.). Dans un contexte concurrentiel aigu, on 
comprend les enjeux managériaux à adapter les prestations de services aux consommateurs qui traversent ces transitions.

L'article s'articule de la manière suivante : le cadre théorique de la recherche portant sur la consommation en période de transition de vie permet de préciser l'objectif de la recherche qui consiste à identifier ce qui dans les expériences de service permet d'aider - ou pas - les consommateurs en transition de vie à (a) diminuer le stress ressenti, et/ ou (b) faciliter la transition de rôle social. Dans un second temps, la méthodologie de la recherche présente la transition du deuil comme terrain d'investigation. Cette transition de vie est particulièrement stressante et constitue ainsi un point d'observation particulièrement pertinent. Dans un troisième temps, les analyses des discours de vingt-huit personnes en deuil indiquent que ce sont des éléments inhérents aux discours des personnels en contact, aux coordinations des interfaces de services, et aux interactions avec les autres consommateurs qui impactent le stress ressenti et permettent de faciliter la transition de rôle social. A la lumière de ces résultats, nous proposons en fin d'article plusieurs préconisations managériales permettant aux prestataires d'adapter leurs offres de services.

\section{CADRE THEORIQUE : LA CONSOMMATION PENDANT LES PERIODES DE TRANSITION DE VIE}

La littérature marketing s'appuie sur deux approches théoriques complémentaires pour appréhender la consommation en période de transition de vie : l'une par la théorie du stress et l'autre par la théorie des rites de passage.

Dans la première approche, les chercheurs considèrent que les transitions de vie suscitent du stress. Ce dernier est provoqué par l'appréciation positive et/ou négative de l'évènement à l'origine de la transition et par la présence d'émotions. Afin de reprendre le contrôle sur leur vie, les individus vont alors déployer un ensemble d'efforts comportementaux (actions) et cognitifs (processus de pensée) visant à réduire ou tolérer ces sources de stress (Lazarus et Folkman, 1984). Ces mécanismes d'adaptation vont directement ou indirectement modifier les comportements de consommation (Andreasen, 1984). Outre les nouvelles consommations (i.e : une personne va consommer des produits de puériculture lorsqu'elle devient parent), la littérature insiste davantage sur le rôle symbolique joué par les produits/marques et pratiques de consommation pour faciliter la transition et réduire le stress. Par exemple, une personne qui vient de divorcer peut changer de style, rejeter les marques de son ex-conjoint ou modifier ses pratiques de consommation dans le but d'affirmer sa nouvelle autonomie (McAlexander, Schouten et Roberts, 1993). La retraite est une autre transition de vie importante pendant laquelle la consommation va servir de facilitateur d'adaptation. Ainsi Schau et al (2009) ont constaté la résurgence des projets de vie de la post-adolescence lors de la transition de la retraite. Ces liens sont à considérer simultanément au capital humain et social dont l'individu dispose. Le capital humain est constitué de l'ensemble des expériences, ressources, qualifications, compétences et connaissances accumulées tout au long de la vie, mais également des soutiens sociaux dont l'individu dispose. En effet, ce capital, parce qu'il façonne les comportements et les pensées, joue sur l'adaptation aux transitions de vie (Moschis, 2012). Le capital social pour sa part, fait référence à la symbolique des rôles sociaux et souligne que les individus modifient leurs comportements, afin de répondre aux attentes sociales prescrites dans les nouveaux rôles à assumer.

La seconde approche mobilisée en marketing, s'appuie sur la théorie des rites de passage (Van Gennep, 1960), selon laquelle tout changement de place, de statut, de position sociale ou d'âge s'opère selon une structure tripartite (détachement / liminalité / intégration), rythmée par des rites. La liminalité définit cette période particulière d'entre deux statuts, une période 
de marge dans laquelle les individus sont détachés de leur situation précédente mais pas encore intégrés dans la nouvelle. Les rites sont des séquences d'action stéréotypées, des modes d'actions déterminés, qui remplissent des fonctions sociales et des fonctions identitaires. Comme le souligne Durkheim (2003), participer au rite c'est montrer son appartenance au groupe. Le rite a un effet curatif et il libère du besoin de réfléchir. Si les sociétés et cultures primitives prescrivaient les rites de passage, il revient aux individus dans les sociétés contemporaines de se définir par et pour eux-mêmes des manières d'agir lors des transitions de vie. Ils vont ainsi construire leurs propres rites de passage en puisant dans la culture les symboles et activités disponibles (Metha et Belk, 1991 ; Schewe et Balazs, 1992), la consommation en est une. Shouten (1991) met ainsi en évidence que la consommation de chirurgie esthétique permet aux personnes en transition de vie de reprendre le contrôle de leur corps et de leur destinée. De la même manière, les décisions relatives aux possessions protègent le groupe déstabilisé par la mort de l'un de ses membres et permettent à l'individu de garder prise sur cet aspect de son existence (Pavia et Mason, 2004).

Ainsi la littérature sur les consommateurs en transition de vie se focalise principalement sur les modifications du comportement suite aux évènements de vie et/ou au rôle symbolique que le produit ou la marque peuvent jouer pour soutenir le consommateur dans leur transition de vie. Les postes de consommation les plus investigués par les recherches sont les possessions (Noble et Walker, 1997 ; Hopkins et al, 2014). Les activités de services sont plus largement ignorées, elles concernent les prestations contraintes par la transition (i.e. Plaud, 2014 ; les services à la personne ou les funérailles dans le cas d'un décès par exemple) ou encore celles liées aux altérations physiques (i.e. Shouten, 1991; coiffure / chirurgie esthétique). Dans tous les cas, les recherches se focalisent sur l'approche en termes de sens donné à la consommation et/ou au processus de désengagement des services. Les expériences de services telles qu'elles sont vécues et perçues par les consommateurs en transition de vie sont absentes de ces recherches.

Pourtant, il est particulièrement intéressant de se focaliser sur ce point car tant l'identité personnelle que l'identité sociale des consommateurs s'expriment pendant les expériences de service (Goodwin et Gentry, 1997). Au cours des services, le consommateur expérimente une série de moments au cours desquels il interagit avec les éléments mis en place par le prestataire de service. Ces interactions recouvrent tant les relations du consommateur avec les personnels, qu'elles soient directes (en face à face) ou indirectes (via une interface), que les relations des consommateurs entre eux. Grönross (1988) qualifie ces interactions de «moments de vérité », car ils sont au cœur des expériences de service et vont avoir une influence sur le bien-être des consommateurs. Dans un tel contexte, notre objectif est d'identifier ce qui dans les expériences de service permet d'aider - ou pas - les consommateurs en transition de vie à (a) diminuer le stress ressenti, et/ ou (b) faciliter la transition de rôle.

\section{METHODOLOGIE DE LA RECHERCHE : LA TRANSITION DU DEUIL COMME TERRAIN D'INVESTIGATION}

Le terrain d'investigation de cette recherche porte sur la transition du deuil. Ce dernier fait l'objet d'un intérêt particulier en comportement du consommateur (Plaud et Urien, 2016) et est considéré comme la transition la plus stressante de l'existence (Holmes et Rayes, 1967). On peut donc considérer que cette transition constitue un point d'observation particulièrement pertinent. D'autant plus que le stress est d'autant plus fort que la transition de vie n'a pas été anticipée (Fellerman et Debevec, 1992) En outre, sous l'effet d'un bouleversement des 
équilibres démographiques à l'horizon 2020-2030, le deuil va concerner de plus en plus de nos contemporains (Clavandier, 2009).

Des entretiens ont été menés auprès de 28 personnes âgées de 58 à 81 ans. Le critère homogène pour la constitution de notre échantillon était d'avoir perdu un proche depuis plus d'un an et moins de 4 ans. Cet intervalle de temps est notamment justifié pour des raisons déontologiques (Plaud, 2014). Au final, notre échantillon est composé exclusivement de femmes, veuves de leur conjoint (tableau 1). Le veuvage à un âge avancé est une expérience datée et sexuée : 88\% des personnes veuves en France sont des femmes de plus de 60 ans (INSEE). Toutes les personnes rencontrées (sauf une, Karine) ont des enfants. La présence d'enfant ne constitue pas, sur ce volet précis des expériences de services, un élément déterminant pouvant nuancer les ressentis exprimés.

Ces entretiens ont évolué d'une posture non directive à une posture semi-directive. Le guide d'entretien démarre par une question générale invitant la personne à dire ce qui a changé dans sa vie depuis le décès du conjoint (parlez-nous de l'événement de vie ; parleznous des difficultés rencontrées depuis cet évènement / relances sur les démarches à conduire, l'impact sur leurs habitudes). Puis les thèmes abordés se centrent sur les expériences de services et les difficultés rencontrées (qu'est-ce qui vous a marquél satisfait ou non ? relances sur les services quotidiens et les services liés à la transition de vie). Nous avons pris soin à ce que les personnes nous racontent comment les expériences se sont passées de leur point de vue. La taille de notre échantillon a été déterminée selon le principe de saturation théorique.

Tableau 1 - Descriptif de l'échantillon

\begin{tabular}{|c|c|c|c|c|c|c|c|}
\hline Nom & Age & CSP & Deuil & Nom & Age & CSP & Deuil \\
\hline Aida & 63 & $\begin{array}{c}\text { Profession } \\
\text { intermédiaire }\end{array}$ & Parent & Jeanine & 74 & $\begin{array}{l}\text { Agriculteur, } \\
\text { exploitant }\end{array}$ & Conjoint \\
\hline Annette & 68 & $\begin{array}{c}\text { Employée, } \\
\text { ouvrier }\end{array}$ & Conjoint & Jeannette & 79 & $\begin{array}{l}\text { Agriculteur, } \\
\text { exploitant }\end{array}$ & Conjoint \\
\hline Antoinette & 65 & $\begin{array}{l}\text { Employée, } \\
\text { ouvrier }\end{array}$ & Conjoint & Jocelyne & 74 & $\begin{array}{c}\text { Employée, } \\
\text { ouvrier }\end{array}$ & Conjoint \\
\hline Béatrice & 65 & Femme au foyer & Conjoint & Karine & 65 & $\begin{array}{l}\text { Employée, } \\
\text { ouvrier }\end{array}$ & Conjoint \\
\hline Catherine & 71 & Commerçant & Conjoint & Line & 58 & $\begin{array}{l}\text { Employée, } \\
\text { ouvrier }\end{array}$ & Conjoint \\
\hline Claire & 76 & Femme au foyer & Conjoint & Marie & 65 & $\begin{array}{l}\text { Employée, } \\
\text { ouvrier }\end{array}$ & Conjoint \\
\hline Denise & 68 & $\begin{array}{c}\text { Employée, } \\
\text { ouvrier }\end{array}$ & Conjoint & Marion & 67 & Commerçant & Conjoint \\
\hline Edith & 72 & Commerçant & Conjoint & Martine & 81 & Femme au foyer & Conjoint \\
\hline Elise & 65 & Commerçant & Conjoint & Nathalie & 79 & $\begin{array}{c}\text { Employée, } \\
\text { ouvrier }\end{array}$ & Conjoint \\
\hline Eloise & 75 & $\begin{array}{l}\text { Employée, } \\
\text { ouvrier }\end{array}$ & Conjoint & Paulette & 74 & $\begin{array}{c}\text { Profession } \\
\text { intermédiaire }\end{array}$ & Conjoint \\
\hline Evelyne & 61 & $\begin{array}{l}\text { Employée, } \\
\text { ouvrier }\end{array}$ & Conjoint & Simone & 65 & Commerçant & Conjoint \\
\hline Geneviève & 72 & $\begin{array}{c}\text { Employée, } \\
\text { ouvrier }\end{array}$ & Conjoint & Sylvie & 63 & $\begin{array}{c}\text { Profession } \\
\text { intermédiaire }\end{array}$ & Parent \\
\hline Gisèle & 67 & $\begin{array}{c}\text { Profession } \\
\text { intermédiaire }\end{array}$ & Conjoint & Thérèse & 81 & Femme au foyer & Sœur \\
\hline Jacqueline & 75 & Employée & Enfant & Yvonne & 64 & Commerçante & Conjoint \\
\hline
\end{tabular}

Les entretiens ont duré entre 30 minutes et 4 heures 30 et se sont déroulés au domicile des personnes interviewées. Ils ont été enregistrés avec l'accord des participants et intégralement 
retranscrits par les auteurs. Les données collectées représentent 900 pages de retranscription ce qui correspond à 54 heures d'entretien cumulées. Les services qui ont été abordés par les répondants ont été les services habituels (banque et administration par exemple) ou hédoniques (les voyages par exemple) ainsi que les prestations de services dont l'usage a été contraint par le décès (service funéraire).

Une analyse thématique des données a été effectuée. La grille de lecture était d'identifier ce qui dans les expériences de service avait un impact sur le stress et la transition de rôle social. Dans un premier temps, les verbatim ont été codés dans des thèmes indiquant la teneur des propos. Puis, nous avons travaillé selon une méthode ascendante, c'est à dire que les thèmes partageant une notion commune ont été regroupés dans des catégories plus générales qui ont progressivement émergé. A l'issue de ces traitements et dans la continuité de la problématique abordée ici, nous avons retenu quatre catégories d'analyse : les discours explicatif et émotionnel du personnel en contact, la coordination des interfaces de service et les relations avec les autres consommateurs. Afin d'améliorer la validité des résultats, un travail de double codage a été réalisé par les auteurs sur une partie de notre corpus.

\section{RESULTATS ET DISCUSSION}

Sur la base des analyses conduites nous mettons en évidence d'une part que la nature des interfaces de service ainsi que les éléments de contexte (décors, théâtralisation, etc.) n'apparaissent pas comme déterminant des expériences de service. D'autre part, ce qui joue un rôle clé relève du personnel en contact et des autres consommateurs. Durant la transition du deuil, ces points sont autant de moments de vérité (Grönross, 1988) qui ont un impact sur le ressenti des consommateurs. Ainsi, quatre éléments des expériences de services ont un impact positif et/ou négatif sur le stress ressenti, ainsi que sur la facilitation dans la transition de rôle: (a) le discours explicatif et (b) émotionnel du personnel en contact; (c) la coordination des interfaces de services; (d) et les relations avec les autres consommateurs. Afin de mieux situer les enjeux pour les prestataires, nous avons resitué ces éléments dans le schéma de la servuction d'Eiglier et Langeard (1987) - (Figure 1).

Figure 1. Les expériences de services dans le processus de servuction 


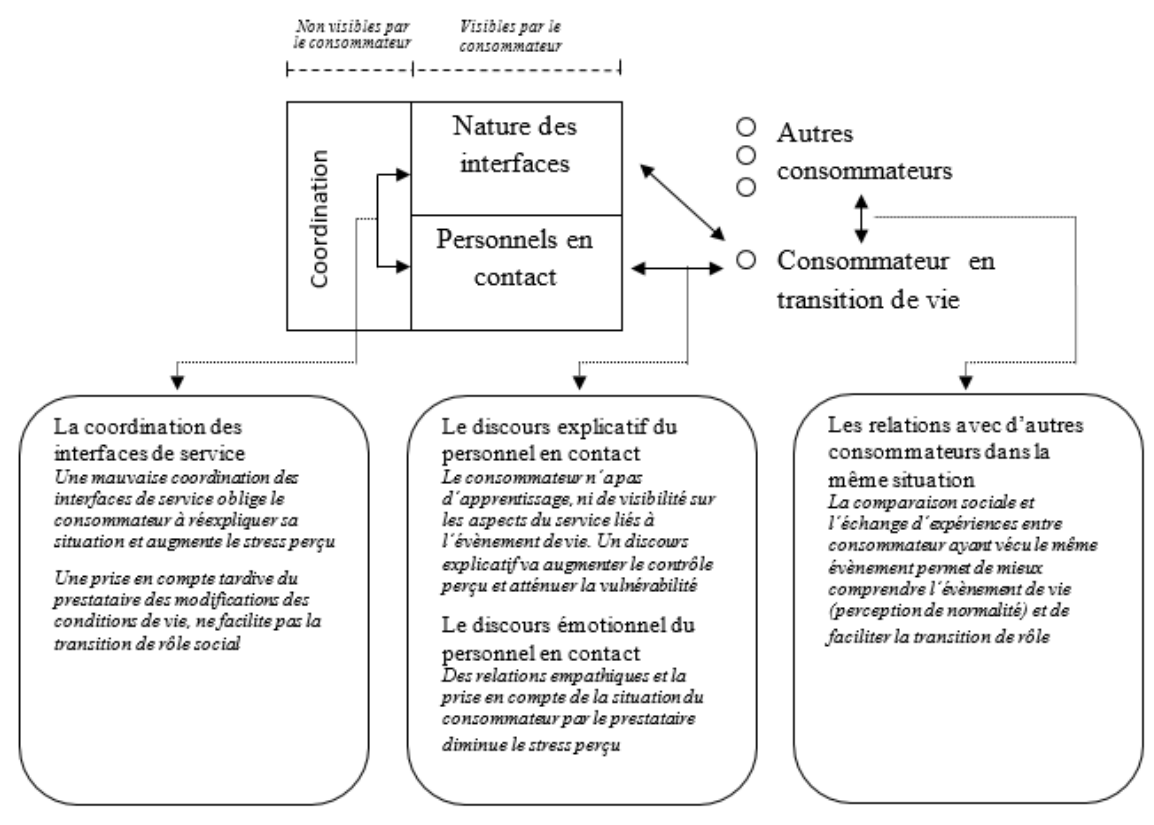

\section{3-1. Le discours explicatif du personnel en contact : un soutien social nuancé}

Dans la continuité des travaux sur le deuil (Gentry et alii, 1995; Plaud, 2014), les personnes que nous avons rencontré expérimentent une situation de stress et de vulnérabilité, dont l'intensité et le vécu est néanmoins propre à chaque individu. Pour autant, elles sont tenues de conduire des démarches pour le moins pénibles mais incontournables, ceci afin d'actualiser leur situation auprès de divers prestataires de services; qu'ils soient institutionnels (caisse de retraire, impôts, etc.) ou commerciaux (banque, etc.).

Nos répondantes expriment en premier lieu leur non connaissance de certaines démarches. Si celles relatives à la prise en charge du défunt semblent a posteriori d'évidence, d'autres sont inconnues et se révèlent complexes :

\footnotetext{
"On a tellement de papiers à faire!, tellement, tellement, il faut changer un tas de trucs, il faut changer la carte grise, il faut changer toutes les assurances, mettre, heu, à mon nom au lieu que ce soit au nom de mon mari et puis des trucs comme çà, un tas, un tas de choses! oui, comme l'électricité, le téléphone des tas de trucs comme çà, il faut amener des certificats de décès partout hein ». (Edith)

«Il faut savoir ce qu'on a droit parce que ce n'est pas automatique. Par exemple, comment, après la mort, il fallait faire une déclaration, y avait une assurance vie, je n'ai pas su et le délai était passé » (Claire)
}

Ces témoignages font écho aux travaux de Gentry et alii. (1995). Selon eux, les consommateurs en deuil sont confrontés à des situations pour lesquelles le processus d'apprentissage est inopérant, ce qui les laisse désemparés et les rend vulnérables face au marché.

En second lieu, si les consommateurs en deuil «ne savent pas », ils pourraient trouver dans le marché les ressources nécessaires pour combler leur méconnaissance. Ce n'est pas toujours le cas. En effet, nos données mettent en évidence le manque d'explications de la part des prestataires, voire leur incapacité à les aider dans leur nouvelle situation, ce qui accentue la 
perte de contrôle de l'environnement (Jeannette) et parfois crée un sentiment d'injustice (Annette) :

«Je reçois des impôts à payer tout de suite, sous 5 jours, mais c'est pas possible, deux fois 63 et 66 euros! [...]. Une fois que c'est payé, je suis tranquille, [mais plus tard] j'ai eu 180 euros à payer aussi, je dis mon Dieu mais qu'est-ce que ça veut dire tout ça? [Et la banque elle ne vous a pas aidé dans les papiers?] Ha si mais elle ne savait pas toujours ce qu'il fallait faire non plus et puis moi j'étais paumée » (Jeannette)

«Comme dit le notaire, si vous payez pas pour le 15 novembre, où je sais plus quand, pour fin novembre, vous serez pénalisée. J'dis comment? pénalisée? J'ai dis "je vous donne une somme comme çà [et en plus] vous allez me pénaliser! »(Annette).

Il semble délicat de démêler dans ces témoignages ce qui relève d'un ressenti négatif lié à la douleur du deuil, d'une réelle incompétence des prestataires, ou encore des effets de classe sociale dans les ressentis du rapport aux administrations. En effet, comme le met en évidence Siblot (2005), «les relations aux administrations prennent des traits spécifiques dans les classes populaires » (ce qui est le cas d'Annette et Jeannette), marquées notamment par des formes de domination culturelle et symbolique. Néanmoins, si l'on se place du point de vue du client, force est de reconnaitre que dans des situations de vie délicates, les défaillances des prestataires aiguisent le stress des usagers (Quilliam, 2008). Nos répondantes expriment ainsi, à leur manière, la sensation de perdre le contrôle de leur situation. Alors que Jeannette est "paumée » et qu'Annette n'en "peut plus », elles ne trouvent pas auprès de prestataires spécialisés l'aide dont elles ont besoin.

En troisième lieu, pour affronter les démarches à conduire et faire face à ce déficit de contrôle, le soutien venant des proches est particulièrement apprécié. Précisons que ces deux femmes ont chacune un enfant mais que ni l'une ni l'autre ne les évoquent comme un soutien mobilisable dans cette situation. Antoinette par exemple sait qu'elle peut compter sur sa bellesœur comptable, donc perçue comme experte en «déclarations »:

"[ vers qui vous vous êtes tournée ?] C'est ma belle-sœur, elle était comptable chez elle donc elle a l'habitude de faire les déclarations, elle m'a dit tu fais comme çà bon bah, cette année y aura pas de problème à le faire comme je suis toute seule, j'aurai qu'une feuille » (Antoinette).

Même situation pour Geneviève dont la nièce est comptable également. Dans le désordre du deuil qu'elle vit, le soutien que lui apporte sa nièce est «une source dans le désert». D'autres répondantes se sont tournées vers les professionnels :

\begin{abstract}
«Je suis pas tellement douée, et bah j'allais avec tous les papiers, on a commencé à aller chez le notaire, les papiers il les a fait, le notaire a fait tout, il a tout fait, c'est pour çà je dis heu, comme çà moi je serai sûre que ce sera bien fait » (Nathalie).

"Et bah vous savez des gens des fois qu'on dit, ah là, là, là !, par exemple les impôts, ha oui, ha! j'ai été aidé d'une façon formidable aux impôts, je suis tombée sur deux femmes, j'ai été deux fois, deux ou trois fois et à chaque fois c'est des gens vraiment charmants qui m'ont bien aidé, qui m'ont aidé, dirigé, tout dans les services qu'il fallait, faire comme ceci, faire comme cela» (Simone).
\end{abstract}

Enfin, certaines personnes que nous avons rencontré soulignent qu'elles n'ont eu besoin de personne, ni d'aide de quelque nature que ce soit : "Moi j'ai fait tous mes papiers tranquille, toute seule » nous a confié Paulette ; même cas pour Catherine. Au-delà, ou en sus de la situation de deuil, se dessinent ici de nouveau des différentiels relatifs à la classe sociale. 
Catherine (ancienne gérante) comme Paulette (ancienne profession intermédiaire) disposent toutes deux d'un capital culturel et symbolique leur permettant sans doute d'aborder plus sereinement le volet administratif du deuil.

Les témoignages recueillis illustrent toutes les nuances possibles dans le soutien que les prestataires sont susceptibles d'apporter aux personnes en deuil. En effet, si dans le cas de Nathalie, c'est le résultat du service qui est perçu comme positif, le cas de Simone met en évidence que c'est le personnel en contact qui cristallise des ressentis positifs, en dépit pourtant d'avoir eu à se rendre à plusieurs reprises sur place. Pour elle, l'aide des professionnels est un complément, tandis que pour Nathalie, les prestataires ont tout pris en charge. Guider le consommateur dans une période particulièrement critique de son existence, lui faciliter les démarches sont donc une manière d'accompagner les consommateurs, ce qui in fine atténue le stress généré par la transition de vie (Goodwin et Gentry, 1997).

\section{3-2. Le discours émotionnel du personnel en contact : une attitude souvent compréhensive}

En sus de l'aspect explicatif du discours ci-avant explicité, la perception de «perdre pied » suite à l'évènement de vie peut être atténuée par la capacité du personnel à tenir compte des modifications dans la situation, à comprendre les difficultés et à s'y adapter. Le cas de Geneviève, en découvert bancaire suite au frais liés à l'enterrement de son mari, illustre notre propos :

\footnotetext{
«Moi je m'imaginais pas, je sais pas si vous savez, le prix des enterrements! En prenant le premier prix dans tout, [...] en prenant le cercueil le moins cher, en prenant heu, [...] le ras des pâquerettes, [...] moi je savais pas, je savais pas !, Je me suis dis que je vais faire un prêt bon bah voilà, c'est mon amie qui m'a téléphoné «on va te passer de l'argent, tu vas pas faire un crédit pour çà! ». Finalement L. [le prestataire] a compris. J'ai payé en 4 fois, vraiment la qualité des gens de L. est remarquable, vraiment, c'est malheureux de dire çà, une qualité de respect

Et puis après ça, j'étais en débours, j'avais plus de finances, j'étais en débours à la banque! [...] Avec ma carte bancaire je ne pouvais plus sortir d'argent! Rendez vous compte! j'ai toute ma tête mais une personne âgée, n'a plus qu'a se tuer! [...] Heureusement que la banque a été compréhensive et qu'elle a compris » (Geneviève).
}

La compréhension des prestataires et leur réactivité n'a certes pas résolu le problème de Geneviève, mais cela n'a pas ajouté de stress supplémentaire à sa situation. Par ailleurs, on comprend à travers son propos, qu'elle garde un ressenti positif sur ces services (banque et prestataire funéraire). Ainsi, en plus d'être guidés, les consommateurs en situation de vulnérabilité ont besoin d'être compris. En cela, si la consommation apparait comme une stratégie possible de gestion du stress (Mathur, Moschis et Lee, 2008), l'expérience de service à travers l'attitude du personnel en contact, agit sur le stress des consommateurs et lui apporte un soutien émotionnel quand il en ressent le besoin.

L'attitude compréhensive du personnel en contact se niche également dans les interactions du quotidien. Nos données mettent ainsi en évidence que les personnes en deuil apprécient lorsque le personnel en contact comprend les difficultés et prend le temps de demander des nouvelles. Marion par exemple se remémore avec plaisir l'attitude empathique du «monsieur de l'EcoMarché »:

«Ce monsieur qui connaissait mon mari, me demande toujours si j'ai besoin d'aide, de quelque chose. Je ne sais pas, il a l'air de s'intéresser à mon sort. Je ne sais pas, je trouve que c'est important $\gg$ (Marion). 


\section{3-3. La coordination des interfaces de service : un contrôle perçu fragile}

Un autre aspect des services est en jeu lors d'une transition telle que le deuil : celle de la coordination des différents prestataires. D'une part, nos données mettent en évidence que répéter la même démarche auprès de plusieurs prestataires de service a pour effet d'augmenter la douleur et le stress :

«Tout le monde vous tombe dessus, les bureaux et ceci, y faut ce papier ci, il faut celui-là, ah non vous n'avez pas de rendez-vous vous revenez demain, vous revenez après-demain. Combien de fois j'ai pété les plombs dans les bureaux moi! » (Annette)

«Les démarches après bah faut écrire à tout, les maisons de retraite, caisses de retraite, la sécu, la, faut écrire à tous ces organismes-là » (Elise)

Parfois le déficit de coordination peut être imputé à un seul et même prestataire :

"Comme la maison mère était à B., alors là, je téléphonais à $V$. moi. on m'dit ah non c'est pas nous maintenant tout est réglé, c'est parti à B., à la maison mère. Je téléphonais là-bas, oh non c'est pas ici, c'est à $V$. madame. Oh non, mais j'ai, ah non mais là je pouvais plus, ah je pouvais plus, je pouvais plus et c'était toujours çà, partout où vous allez c'était la même chose. On vous dit des fois faut attendre, faut aller, faut payer ceci, faut payer cela, mais fallait payer mais fallait toujours attendre pour avoir les renseignements, les papiers pour ceci pour cela oh! »(Annette)

D'autre part, la non reconnaissance du changement de statut renvoie l'individu à son chagrin et son deuil :

«Il y des frais de dossier à la banque soit disant pour vous accompagner, la banque prend 180 euros et c'est vous qui faites les démarches là, [...] et puis les erreurs y en a, après on reçoit des courriers toujours au nom de monsieur, c'est, c'est un combat après encore ». (Elise)

Dans le cas d'Elise, le rappel de cet évènement constitue un «combat » supplémentaire qui ne permet pas d'investir et d'accepter l'évènement de vie et donc d'investir le nouveau rôle social. Comme le rappelle Quiliam (2008), ces inconséquences des prestataires ne seraient finalement que des détails et des fautes d'inattention, sauf que pour les consommateurs, elles ajoutent de la douleur à la douleur et in fine présentent d'importants coûts cachés pour les prestataires. Parmi ces derniers, rappelons ce constat sans appel : 70\% des veufs et veuves changent de conseiller financier dans les trois mois qui suivent le décès du conjoint (Florian, 2011).

3-4. Les interactions avec les autres consommateurs : recherche de normalité et transitions de rôle

Nos données ont enfin mis en évidence un dernier élément : l'importance des relations entre consommateurs. En effet, les comparaisons directes ou indirectes avec les autres usagers vont avoir une influence sur la perception de normalité et permettre de faciliter - ou non - la transition de rôle. Les comparaissons indirectes renvoient une image sociale et permettent à la personne d'évaluer sa situation comme «normale » ou non «normale ». Prenons l'exemple d'Annette qui depuis le décès de son mari estime que "rien n'est fait pour les femmes seules », elle illustre ses propos par son expérience des voyages : 
«Et puis y a pas grand-chose de fait non plus parce que quand vous partez 3, 4 jours, les chambres c'est toujours à deux lits et c'est plus cher à deux personnes, une personne bah vous payez le même prix que deux, alors c'est pas normal non plus que les hôtels font çà. » (Annette)

Dans son propos, Annette ne se sent pas à sa place dans un univers de service conçu pour des couples, elle évoque même l'idée d'organiser des prestations de voyages dédiées aux veuves. Cette idée de prestations de services dédiées n'est pas dénuée de sens et fait ses preuves. En effet, des prestataires de service - tels que les caisses de retraites, les assurances, en sus de leurs prestations principales, offrent des prestations de confort, comme des sessions d'information sur le veuvage :

\footnotetext{
"C'est vrai que je rejoins, des fois y a des réunions de veuves, femmes et bah c'est vrai que chacune apporte comment elle a pu s'en sortir c'est vrai, elle dit bah moi je suis restée longtemps à la maison mais à la fin je me suis dis bah je vais quand même pas restée ici »(Jeannine).
}

Parmi nos répondants, cinq ont participé à une session sur le deuil organisée par leur caisse de retraite. Si cette session recouvre des objectifs variés, force est de constater que la prise de rôle «veuve » s'en est trouvé «facilitée » pour elles. En effet, quand Gisèle évoque ce moment, c'est le « nous » qu'elle emploie pour marquer son intégration dans un groupe : celui des personnes veuves. Ce «nous » est ce qui vient également quand Annette se remémore comment elle a réagi quand ce stage lui a été proposé : «c'est quand même bien, pour une fois qu'on s'intéresse à nous». Pour ces femmes la parenté d'expérience ressort systématiquement à l'évocation de cette session. Cette parenté permet de se comparer, de savoir si ses propres réactions sont «normales » ou pas, de se constituer de nouveaux amis « comme soi ». L'échange avec les autres participants ainsi que les « cours » dispensés par le psychologue qui encadre la session, permettent d'acquérir les codes et usages des personnes endossant le rôle de veuve:

\begin{abstract}
"J'étais très contente de ce stage là parce que, il a mis au clair des phénomènes physiques inhérents au deuil que j'imaginai, parce que c'était dans le stress mais il les a mis en détail et ça $m$ 'a fait du bien parce que je me suis dit que je devenais pas folle »(Geneviève).
\end{abstract}

Ces résultats prolongent ainsi les travaux conduits sur la vulnérabilité et la recherche de normalité (Baker, 2006). En effet, cette dernière s'encastre dans les expériences de services, via les interactions entre consommateurs. Dans le cas du deuil, la vulnérabilité serait ainsi contextuelle et la rencontre de personnes dans la même situation que soi permettrait aux individus de mieux passer le cap de la transition, pour s'approprier leur nouveau rôle.

\title{
4. IMPLICATIONS MANAGERIALES
}

A l'appui de ces résultats, nous formulons trois préconisations visant à optimiser les expériences de services pour les consommateurs en transition de vie: (1) repenser la coordination des interfaces de service ; (2) élaborer des scripts de services pour le personnel en contact ; et (3) et penser aux regards croisés entre consommateurs.

\section{4-1. Repenser la coordination des interfaces de service pour s'adapter aux nouveaux rôles et statuts des consommateurs}

Nos résultats ont montré que la coordination des interfaces de service est un élément important permettant aux consommateurs de mieux vivre les transitions de vie. Nous 
préconisons donc aux manageurs d'engager un processus de centralisation des données administratives et sociodémographiques des individus consommateurs qui peuvent prendre la forme, lorsque cela est possible, d'une coopération avec les autres entreprises concernées, et d'une refonte des procédures standardisées qui n'ont pas été pensées pour les consommateurs en transitions de vie.

Coopération entre entreprises pour les démarches administratives. Nos données montrent que les prestations de services, notamment celles contraintes par un évènement de vie (démarches administratives, assurances, par exemple) peuvent avoir un impact négatif sur les personnes qui se sentent désemparées et parfois dévalorisées par les démarches à accomplir. Un système de "guichet unique" se révèle alors opportun afin de faciliter des démarches parfois longues et complexes. L'initiative des pouvoirs publics quant à la création d'un compte personnel qui centralise les démarches va dans ce sens mais se destine aux détenteurs d'internet. Des mairies, par exemple celle d'Antibes (06), ont également développé un guichet unique à destination des familles avec enfants afin de simplifier les procédures d'inscription à l'ensemble des activités péri et extrascolaires. Les exemples existent donc, reste à les dupliquer pour des cibles et des usages différents. Ainsi, des services publics et/ou des entreprises complémentaires répondant à un même besoin pourraient s'associer pour présenter une interface unique. Appliqué au cas de notre recherche, un guichet «centralisation des démarches suite à un décès » se révèlerait pertinent.

Déstandardiser certaines procédures. Si les démarches ne peuvent pas être centralisées, il convient d'éviter autant que possible les procédures standardisées. Nos résultats doivent attirer l'attention des manageurs sur ce point: l'objectif est de trouver un équilibre entre standardisation, pour augmenter l'efficacité et la rentabilité du service, et procédures à caractère humain. Par exemple en instaurant des mot-clés comme « décès » qui nécessite alors une autre procédure et de reprendre le contrôle humain. Bien souvent en effet, l'impératif de rentabilité est ce qui guide les prestataires qui proposent des services standardisés et sans contact personnalisé (e-service par exemple). Comme le souligne Deyfus (2007) « l'impératif de rentabilité n'a pas pour but ultime d'accroitre la satisfaction des usagers, mais bien de réduire les coûts supportés par l'employeur ». Pour autant, nos résultats plaident pour une personnalisation des services tenant compte à la fois de la transition de vie des consommateurs (dans notre cas, le deuil) mais également de la classe sociale d'appartenance.

\section{4-2. Elaborer des scripts de services pour le personnel en contact pour soutenir les consommateurs en transition de vie}

Nos résultats montrent que le discours du personnel en contact, tant dans ses dimensions explicative qu'émotionnelle, a une influence déterminante sur le ressenti du consommateur en transition de vie, ainsi que sur son évaluation du service. Pourtant si la plupart des prestataires forment leur personnel en contact aux tâches opérationnelles, ils les laissent bien souvent gérer seuls les aspects relationnels. Ainsi les discours peuvent varier en fonction du niveau d'éducation, de l'intuition et de la sensibilité de chacun (Eiglier et Langeard, 1987). Une formation du personnel paraît nécessaire pour leur faire prendre conscience de l'impact potentiel de leur actes et discours sur leur clientèle. Car, comme le rappelle Quiliam (2008), ces inconséquences des prestataires ne seraient finalement que des détails et des fautes d'inattention, sauf que pour les consommateurs, elles ajoutent de la douleur à la douleur.

Dans la continuité de certains travaux, il convient donc d'inclure les transitions de vie comme variables de segmentation (Schewe et Balazs, 1992 ; Goodwin et Gentry, 1997). Cette 
variable servira à élaborer des scripts permettant de dérouler un discours adapté aux individus en transition de vie. Concrètement, à réception d'un avis de décès par exemple, un script spécifique se met en route : diffusion de l'information au conseiller en charge du client ; appel ou contact client pour s'informer de ses attentes ; proposition d'un rendez-vous pour gérer les volets administratifs suite au décès; vérification de la mise en place, etc. De tels scripts sont susceptibles d'éviter les coûts cachés liés à leur non adaptation au consommateur en transition de vie : multiplication des courriers, inadaptation des prestations, voire, plus grave, perte des clients.

\section{4-3. Penser aux regards croisés entre consommateurs en facilitant leurs interactions}

Nos résultats mettent en évidence que des prestations de services adaptées peuvent faciliter la prise du rôle. Dans cette recherche, nous avons exploré les sessions sur le deuil qui permettent à la personne en deuil de passer du rôle d'épouse/époux à celui de veuve/veuf. Ces services parce qu'ils travaillent sur la similarité des expériences de vie (homophilie selon le terme employé par Gentry et alii, 1995) permettent à l'individu de retrouver sa normalité (Baker, 2006). Notre préconisation est donc d'approfondir la compréhension de ces rôles dans le but de proposer des offres adaptées aux besoins de la cible. Ainsi, en plus des services principaux qui satisfont le besoin du client, les prestataires peuvent imaginer des services périphériques dédiés aux évènements de vie qui y ajoutent de la valeur (Grönroos, 1988). Les entreprises œuvrant dans le secteur de la finance et de l'assurance sont particulièrement concernées, tant les décisions attenant à ces domaines sont liées aux évènements de vie. On peut imaginer des échanges d'expériences entre consommateurs à des moments clés de leur existence : par exemple l'apprentissage du rôle social de parent et des décisions financières attenantes au moment de la naissance du premier enfant ou encore celui de caregiver lorsque la génération pivot a toujours en charge ses enfants mais également ses parents qui perdent progressivement leur autonomie (Guillemot, 2018). A charge pour les prestataires, mais également pour le secteur associatif, de faire connaître et d'être proactif au moment où la personne en ressent le besoin.

\section{CONCLUSION}

Cette recherche permet d'enrichir la littérature sur le rôle de la consommation pendant les transitions de vie. En s'intéressant aux expériences de services, elle s'inscrit dans le sillon de Shouten (1991) et Gentry et al (1995). Les résultats de notre recherche permettent de mieux comprendre comment la transition du deuil impacte les expériences de services contraintes par l'évènement de vie et comment elle modifie celles des services du quotidien. Conjointement, ils mettent en évidence le rôle clé du discours des personnels en contact, de la coordination des interfaces de services, ainsi que des interactions avec les consommateurs dans des situations similaires.

Cette recherche présente quelques limites qui constituent des pistes de recherches intéressantes. Il convient ainsi d'augmenter la validité externe de l'étude avec l'investigation d'autres populations et d'autres transitions de vie. En effet, dans le cadre de cette recherche nous avons rencontré exclusivement des femmes en deuil de leur conjoint. Cette population est très nombreuse, due à l'allongement de la durée de la vie et du différentiel d'espérance de vie entre les hommes et les femmes. Néanmoins, il conviendrait de recueillir le point de vue des hommes et de personnes plus jeunes. Par ailleurs, il serait intéressant d'étendre cette recherche à d'autres évènements du cycle de vie. Une confrontation des transitions de vie a priori positive (i.e. naissance) et celles a priori négative (i.e. décès d'un conjoint), pourrait 
mettre en perspective les résultats de la présente recherche. Une autre piste serait de comprendre comment cette expérience de vie impacte tout ou partie du processus décisionnel. Conjointement, en s'intéressant à l'expérience de vie, un design longitudinal pourrait apporter des éclairages complémentaires sur l'évolution des ressentis au cours du temps.

Enfin, nous avons adopté dans cette recherche le point de vue des consommateurs. Les prestations de services étant un processus d'interactions, il serait riche d'enseignements de compléter ce point de vue par celui du personnel en contact et des dirigeants d'entreprises de services. Par ces regards croisés, nul doute que nous gagnerons en compréhension sur les liens entre bien-être et prestations de services.

\section{RÉFÉRENCES}

ANDREASEN A.R., "Life status changes and changes in consumer preferences and satisfaction", Journal of Consumer Research, n¹1, 1984, p784-794.

BAKER S.M., "Consumer normalcy: understanding the value of shopping through narratives of consumers with visual impairments", Journal of Retailing, n ${ }^{\circ} 82$ (1), 2006, p37-50.

CLAVANDIER G., Sociologie de la mort. Vivre et mourir dans la société contemporaine, Paris, Armand Colin, 2009.

DEYFUS F., «Personnels et usagers des services publics : des relations transformées ?»; Regards Croisés sur l'Economie, n², 2007, p.246-254.

DURKHEIM E., Les formes élémentaires de la vie religieuse, Paris, PUF, Quadrige, 2003 (édition originale publiée en 1912).

EIGLIER P. et LANGEARD E., Servuction. Le Marketing des services, McGraw Hill, 1987.

FELLERMAN T. et DEBEVEC K., "Till death do we part: family dissolution, transition, and consumer behavior", Advances in Consumer Research, n 19, 1992, p514-521.

FLORIAN A., "Ten principles for effective grief support", Journal of Financial Planning, $\mathrm{n}^{\circ} 24$ (2), 2011, p36-38.

GENTRY J.W., KENNEDY P.F., PAUL C. et HILL R.P., "Family transitions during grief: discontinuities in household consumption patterns", Journal of Business Research, n³4 (1), 1995, p67-79.

GUILLEMOT S., "Intergenerational transmission in consumer behaviour: An integrative conceptual framework and future research directions", Recherche et Applications en Marketing (English Edition), n³3 (2), 2018, p93-114.

GOGUEL D'AlLONDAS T., Rites de passage, rites d'initiation. Lectures d'Arnold Van Gennep, Les Presses de 1'Université de Laval, 2002.

GOODWIN C. et GENTRY J.W, "The role of services during consumer transitions", Research in Consumer Behavior, n $8,1997, \mathrm{p} 271-291$.

GRÖNROOS, "Service quality: the six criteria of good perceived service quality", Review of Business, ${ }^{\circ} 9$ (3), 1988, p.10-13.

HOLMES T.H. et RAHE R.H., "The social readjustment rating scale", Journal of Psychosomatic Research, ${ }^{\circ} 11$ (2), 1967, p213-218.

HOPKINS C.D., WOOD C., SIEMENS J. et RAYMOND M.A., "A multi-method investigation of consumer response to marketing activities during life transitions", Journal of Consumer Marketing, $\mathrm{n}^{\circ} 31$ (1), 2014, p39-53.

LAZARUS R. et FOLKMAN S., Stress, appraisal and coping, New York, Springer Publishing Company, 1984.

NOBLE C.H. et WALKER B.A., "Exploring the relationships among liminal transitions, symbolic consumption, and the extended self”, Psychology and Marketing, n¹4 (1), 1997, p.29-49 
MCALEXANDER J.H., SCHOUTEN J.W, et ROBERTS S.D, "Consumer behavior and divorce", Research in Consumer Behavior, n6, 1993, p153-184.

MEHTA R. et BELK R.W., "Artifacts, identity and transition: favorite possessions of indians and indian immigrants to the United States", Journal of Consumer Research, n 17, 1991, p398-411.

MATHUR A., MOSCHIS G.P. et LEE E., "A longitudinal study of the effects of life status changes on changes in consumer preferences", Journal of the Academy of Marketing Science, ${ }^{\circ} 36$ (2), 2008, p234-46.

MOSCHIS G.P., "Consumer behavior in late life: current knowledge, issues, and new direction for research", Psychology and Marketing, n'29 (2), 2012, p.57-75.

PAVIA T.M. et MASON M.J., "The reflexive relationship between consumer behavior and adaptive coping", Journal of Consumer Research, n³1, 2004, p.441-454.

PLAUD C., «Besoins et représentations des veuves en matière de soutien social », Décisions Marketing, $\mathrm{n}^{\circ} 74,2014$, p.53-66

PLAUD C. et URIEN B., «Le deuil originel et métaphorique : état de l'art et voies de recherches en comportement du consommateur », Recherche et Applications en Marketing, $\mathrm{n}^{\circ} 31(2), 2016, \mathrm{p} .62-85$

QUILLIAM E.T., "The often-forgotten non-funeral consumer grief for the grieving", The Journal of Consumer Affairs, $\mathrm{n}^{\circ}$ 42(3), 2008, p.471-477.

SCHAU H.J., GILLY M.C. et WOLFINBARGER M., "Consumer identity renaissance: the resurgence of identity-inspired consumption in retirement", Journal of Consumer Research, ${ }^{\circ} 36$ (2), 2009, p.255-76.

SCHEWE C.D. et BALAZS A.L., "Role transitions in older adults: a marketing opportunity", Psychology and Marketing, ${ }^{\circ} 9(2), 1992$, p85-99.

SCHOUTEN J., "Selves in transition: symbolic consumption in personal rites of passage and identity reconstruction", Journal of Consumer Research, n¹7, 1991, p412-425.

SIBLOT Y., «Les relations de guichet : interactions de classes et classements sociaux », Lien social et politiques, $\mathrm{n}^{\circ} 49,2005$, $\mathrm{p} 183-190$.

VAN GENNEP, Les rites de passage, Picard, 1960 ( $1^{\text {ère }}$ édition en 1909) 